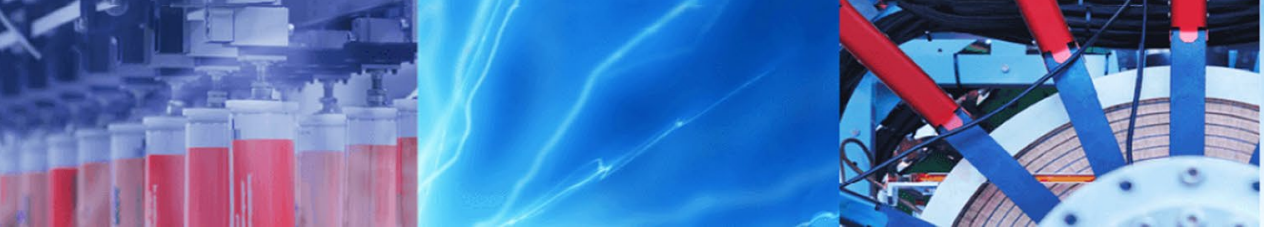

Research Article

\title{
Fair energy efficient scheduler providing high system capacity for wireless networks
}

\author{
Cédric Gueguen ${ }^{1}$ [D $\cdot$ Christopher Merlhe ${ }^{1}$
}

Received: 9 January 2020 / Accepted: 25 November 2020 / Published online: 1 December 2020

(c) Springer Nature Switzerland AG 2020

\begin{abstract}
Guaranteeing high system capacity while offering low system energy consumption remains a crucial issue in wireless networks. This one becomes again more difficult if high fairness and high Quality of Service (QoS) are also required. The simultaneous achievement of these objectives often requires the usage of tradeoffs that generally provide average overall performance. However, researching a static tradeoff on the whole of these criteria is not optimal since the magnitude of importance of these ones vary according to the context. In previous works, a solution has been proposed which highlights that a dynamic trade-off based on system traffic load could outperform the static ones. In underloaded context, the focus should be put on low energy consumption while, on the contrary in overloaded context, priority should be done on high system capacity. Even though efficient, this solution is unable to provide service differentiation and consequently full fairness and adequate QoS in many context particularly when mobile have different delay constraints or throughput requirements. This paper proposes a new access scheme able to provide Fairness, Energy efficiency and high system Capacity that is called FEC scheduler. This scheme heightens the benefits of opportunistic scheduling by extending cross layer technique from physical layer to higher layers. Resource allocation is performed thanks to a system of weights that dynamically accounts for all three: the radio conditions, the system energy profitability to select a specific user and its experienced QoS. Performance evaluation shows that this solution offers efficient global system energy consumption without downgrading the system capacity while enabling good QoS differentiation and fairness.
\end{abstract}

Keywords Wireless network · Opportunistic scheduling · Cross-layer design · QoS · Energy consumption

\section{Introduction}

In wireless networks, radio resources are scarce and limited. In addition, the number of mobile is continuously growing and applications are increasingly demanding about throughput and delay constraints. This requires to heighten uninterrupted efforts to increase spectral efficiency and system capacity as well as possible while ensuring service differentiation and fairness. In addition, user mobility requires to reduce energy consumption in order to extend battery lifetime (which is an important Quality of Experience (QoE) criterion) while also helping to fight against environmental problem and global warming (climate change). The efficiency of radio resource management algorithms are crucial in order to guarantee these key objectives and this topic is well investigated in literature.

Conventional access methods like Round Robin (RR) [1-3] and Random Access (RA) were initially developed for the wired context and are not well adapted to the wireless environment. Since they do not take into account the physical layer information in the allocation process, they provide very poor throughput. Indeed, the major phenomena that affect the quality of the radio transmission are

Cédric Gueguen, cedric.gueguen@irisa.fr; Christopher Merlhe, christopher.merlhe@irisa.fr | ${ }^{1}$ University of Rennes 1, IRISA laboratory, Rennes, France. 


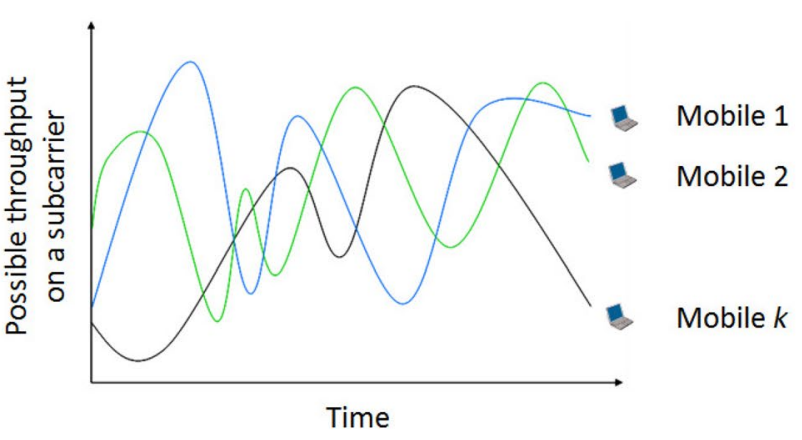

(a) Time-related throughput/Resource Unit (RU) variation due to multi-path fading.

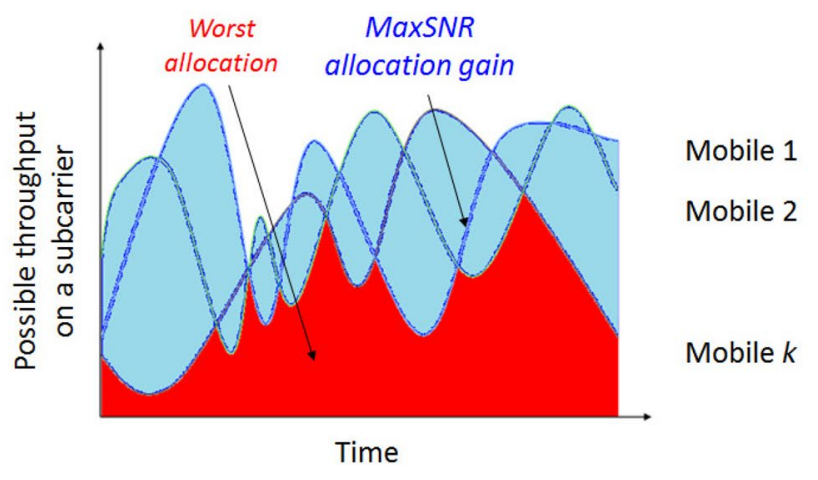

(b) Opportunistic system capacity increase.

Fig. 1 Benefit of opportunistic scheduling strategies on spectral efficiency and system capacity [11]

path loss, shadowing and multipath fading. This widely affects the user throughput with high gaps across the time and high disparities on each subcarrier. This makes a mobile could have strong throughput on a specific frequency at a given time but maybe not to another frequency or instant. This should not be ignored in order to provide high spectral efficiency. Several works have been conduct to develop throughput efficient schedulers using opportunistic approaches that wisely choose which user offers the best profitability in term of bit per Resource Unit $(\mathrm{RU})$.

In $[4,5]$, Maximum Signal to Noise Ratio (MaxSNR) scheduler is proposed. It grants resources to the mobile with the best channel conditions at a the considered time. It takes advantage from frequency and multi-user diversity in order to increase the system throughput (Fig. 1b). However, this scheduler is highly unfair offering disproportionate priority to close user to the access point and is also unable to provide any service differentiation that often conduct to a poor global QoS and QoE.

Proportional Fair (PF) and PF-based algorithms solve this issue [3, 6-9]. The basic principle is to grant resources to a mobile when its radio conditions are the most suitable as regards to its time average. This approach is fairer than MaxSNR about mobiles at different distances since they have statistically an equal probability to access radio resources. In [10], a Weighted Fair Opportunistic scheduler (WFO) is proposed. It outperforms PF scheduling providing better fairness and service differentiation and higher spectral efficiency thanks to a more efficient usage of the multi-user diversity. Nonetheless, all these solutions have an intense deficiency in term of energy management.

Following the objective to provide higher battery autonomy to mobiles while contributing to solve environmental problems, many solutions focusing on energy consumption minimization have been developed. The
Power-based Proportional Fairness (PPF) [12] proposes PF-based scheduler that avoids the inefficient allocations (with low SNR) and delay flows which have significant mean energy consumption. Energy efficiency is lightly increased thanks to an access to the medium given only to mobiles with decent radio conditions. This allows to always use great modulation order which are the most advantageous. However, this also segregate mobiles with high traffic load which use more Resource Unit (RU) and therefore consume more energy. This can potentially damage global system QoS and QoE. Moreover, note that the best strategy to reduce energy consumption is not to optimize the modulation but mainly to increase the users sleep time duration. The solution Opportunistic Energy Aware scheduler (OEA) presented in [13] is designed on this principle. It compresses the active mode of each mobile (i.e. time of activity mobile $=$ transmission time), voracious in energy. This is done thanks to an allocation that tries to obtain good radio condition and short active-sleep mode together (while classical opportunistic schedulers potentially activate several mobiles in a given time slot, OEA limits this number). Radio condition are considered in the OEA allocation process and only allocations with good modulation are conserved. This contributes to avoid low throughput efficient allocation that can be considered as a waste in term of energy while providing high sleep time duration. This strategy can be extended. For instance, the solution T-MAC presented in [14] is close to be an extreme version of OEA. The main directive for this solution is to allocate all the subcarriers by Time Slots (TS) to only one user. This significantly increases the sleep time duration but this is done at the expense of a heavily multiuser diversity benefits decrease leading to lower throughputs. The major drawbacks of these schedulers are their shortage in terms of fairness and a weak provided global system capacity that restricts their usage to underloaded context. 
As optimized system energy consumption should not allow to forget to respect QoS requirements and have high system capacity, other approaches should be designed to bring them together such as: high system capacity, more fairness and energy consumption minimization when possible (if it does not downgrade the mobiles QoE).

Dynamic Trade-off scheduler (DT) has been developed from this analysis [11]:

- In low loaded context, radio resources are plentiful and the system can easily meet user needs. Since guaranteeing high QoS and QoE are easily achievable due to large surplus of available radio resources, the focus should be put on energy rather than system throughput.

- In highly loaded and overloaded system, radio resources are particularly valued and system meets high difficulties to satisfy all users. Since it could consider that satisfying user delay constraints and throughput requirements is more important than reducing energy consumption at all costs, the lack of available $\mathrm{RU}$ requires that schedulers focus on spectral efficiency in order to preserve QoS and reach high system capacity.

Consequently, DT dynamically adjusts its behavior according to the traffic load context. More it detects a surplus of unused radio resources, more it supposes easier the QoS to be guaranteed and DT orients its scheduling strategy to be energy focused (limiting the number of activated mobiles per time slot, increasing the sleep time duration). On the contrary, if DT detects a lack of available radio resources, it orients its scheduling strategy to enhance spectral efficiency in order to withstand the load raise (increasing the number of activated mobiles per time slot, making a better usage of the multi-user diversity). Even though DT is really competitive versus specialized schedulers on theirs scope of usage (versus OEA in underloaded context about energy efficiency and versus MaxSNR in highly loaded context about spectral efficiency), this solution fails to provide service differentiation, full fairness and adequate QoS in many context particularly when mobile have different delay constraints or throughput requirements. In addition, DT adjusts its objectives priority (energy versus system capacity) thanks to the system bandwidth usage ratio that does not take into account the significant importance of flows variabilities and application constraints. This conducts its dynamic trade-off to sometimes be inaccurate (triggered too early while no QoS problem are met or too late when QoS is already widely affected).

In this paper we propose a new scheduler able to provide Fairness, Energy efficiency and high system Capacity (FEC). Taking into account a QoE metric in order to decide of the system objectives priorities (energy minimization or system capacity increase), FEC is able to solve DT inaccuracy focuses. Indeed, even if the bandwidth usage ratio used in DT is a good metric to decide if mobile could expect good QoE (focus could be done on energy) or not (focus must be done on system capacity), it is far to be fully accurate ${ }^{1}$. In our proposal, until it is possible (i.e. as long as mobiles experience acceptable QoE), the focus is conserved on energy minimization. If $\mathrm{QoE}$ experienced by mobiles begins to decrease, FEC proportionally increases the focus on spectral efficiency in order to preserve it. Moreover, the solution is built in a cross layer approach that offers other advantages. Physical layer information allows to make opportunistic scheduling which ensures high system capacity. Higher layer parameters considered with the QoE metric of the FEC allow to provide, in addition, high fairness and QoS differentiation. Performance evaluations show that FEC scheduler provides efficient global system energy consumption close to OEA without harming the system capacity that reaches MaxSNR performance. This is done while enabling good QoS differentiation and fairness close to the specialized scheduler on these metrics (WFO).

This paper is built as follows: Sect. 2 shows the system description, Sect. 3 describes the proposal and Sect. 4 shows performance evaluations. Section 5 is the conclusion.

\section{System description}

This paper studies the radio resource allocation problem for the set of users located in the coverage zone of an access point. A centralized approach is assumed since this allows efficient opportunistic scheduling approaches. The physical layer is considered to operate using a Time Division Duplex mode (TDD) which allows a good compatibility with the Orthogonal Frequency Division Multiplexing (OFDM) based transmission mode [15]. The bandwidth is divided in sub-frequency bands (i.e. subcarriers) and, in time domain, in frames themselves splited in Time Slots (TS) of constant duration (which is an integer multiple of the OFDM symbol duration). Each sub-frequency band is considered such as its width is inferior to the coherence bandwidth of the channel. Moreover, each frame duration is assumed equal to a value inferior to the coherence

\footnotetext{
1 Even at low traffic load, DT can experience difficulties due to mobiles with high service flow variability or/and with strict application delay constraints. At the opposite, even at high traffic load, QoE could stay acceptable due to moderate traffic burst or application delay constraints easy to satisfy. In these two cases, DT will focus on the wrong objective.
} 
time of the channel. This allows the transmission on each subcarrier to experience flat fading during each frame. In addition, full knowledge of the radio conditions is supposed to be available at the receiver [16]. Consequently, access node is able to estimate channel attenuation at a given time on each subcarrier and for each users thanks to SNR measurement of the signal sent by each mobile (for example during the uplink contention subframe). Following [17], channel state can be assumed stable on a scale of $50 \mathrm{~ms}$. This requires that users have to transmit their control information alternatively on each subcarrier once for several frames (in order for the access node to successfully refresh the channel state information).

Each (subcarrier, time slot) pair represents an elementary Resource Unit (RU) which can be allocated to any user with a specific modulation order. Transmissions performed on different RUs by different users have independent channel state variations. On each RU, a modulation order adapted to the channel state (between the access point and the selected mobile) is assumed. This provides the flexible resource allocation allowing opportunistic scheduling.

\section{Fair, energy efficient and high system capacity scheduler (FEC)}

The FEC scheduling algorithm is based on weights that set the dynamic priorities in order to process to efficient radio resources allocation. These weights are designed in order to achieve three important goals which are separately detailed in the following:

- Maximization of spectral efficiency and system capacity,

- Guarantee of fairness,

- Minimization of energy consumption.

Then the algorithm flow chart and the merging of the previous weights in the balanced FEC solution will be presented.

\subsection{System capacity maximization}

A major objective of FEC scheduler is to optimize the system throughput. This is performed in a MAC/PHY opportunistic approach. Data integrity requirements of mobiles are enforced considering each one independently, adapting the modulation and the transmit power to the user specific channel state. On the allocation process of each frame, the radio resource allocation algorithm computes the maximum number of bits $q_{k, n}$ that can be potentially transmitted in a TS $t$ of subcarrier $n$ if assigned to service flow $k$ while guaranteeing to respect its Bit Error Rate target $\left(B E R_{\text {target }, k}\right)$, for all $k$ and all $n[18]$ :

$q_{k, n} \leq\left\lfloor\log _{2}\left(1+\frac{3 P \times T_{s} \times\left(\frac{1}{d_{k}}\right)^{\gamma} \times \alpha_{k, n}^{2}}{2 N_{0}\left[\operatorname{erfc}^{-1}\left(\frac{B E R_{\text {target },}}{2}\right)\right]^{2}}\right)\right\rfloor$,

with:

- $P$ the power of the transmission,

- $T_{s}$ the duration of an OFDM symbol,

- $N_{0}$ the spectral density of noise,

- $d_{k}$ the distance between the access point and the mobile linked to the service flow $k$,

- $\alpha_{k, n}^{2}$ the flat fading experienced by this user on subcarrier $n$,

- erfc the complementary error function such as: erfc $z$ $=1$ - erf $z$ with erf $z$ the error function (also called the Gauss error function) [18].

$\alpha_{k, n}$ is Rayleigh distributed such as its expectation is equal to 1 . Due to multi-path fading, $\alpha_{k, n}^{2}$, the potential number of bit $q_{k, n}$ that a mobile can send (or receive) on a radio resource unit fluctuates over the time. The exponent $\gamma$ corresponds to the experienced path loss and shadowing. It generally varies from 2 to 4 considering environment density level. In the following, in order to consider dense urban context, $\gamma$ is assumed equal to 3.5.

The supported modulation orders are assumed QAM and limited such as $q$ belongs to the set $S=\left\{0,2,4, \ldots, q_{\max }\right\}$. Afterward, the maximum number of bits $m_{k, n}$ that can be transmitted on a time slot of the subcarrier $n$ if this $\mathrm{RU}$ is allocated to the service flow $k$ is:

$m_{k, n}=\max \left\{q \in S, q \leq q_{k, n}\right\}$.

MaxSNR based schemes lead to allocate RUs to the service flow which have the greatest $m_{k, n}$ values. This strategy significantly increases system capacity at a short time scale. Nevertheless, mobiles close to the access point have a better average throughput per Resource Unit (RU) than farthest. This induces that, with MaxSNR scheduler, close mobiles have statistically higher chances to access to the radio resources. Consequently, far mobiles will often obtain radio resources after close users making them overpassing their delay constraints and becoming unsatisfied. To ensure more fairness between users at different positions while preserving the system capacity maximization, a fairness parameter is added in FEC. 


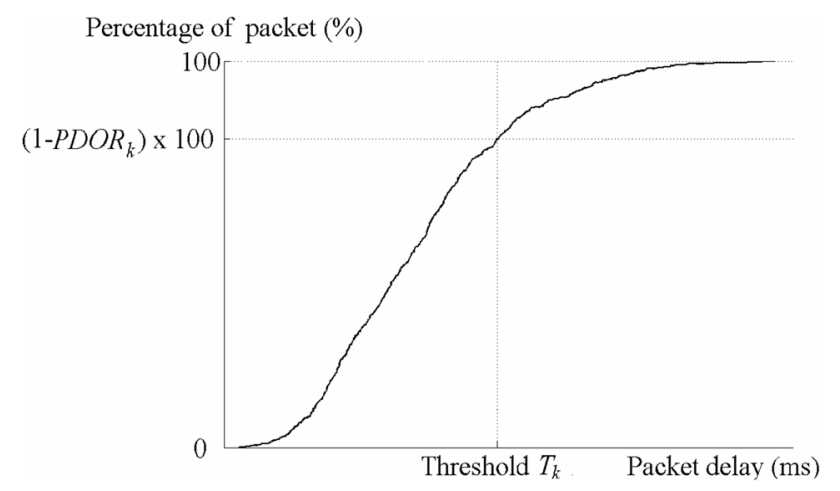

Fig. 2 Example packet delay CDF and experienced PDOR

\subsection{Fairness guarantee}

One of the major goals of the FEC scheduler design is to successfully support all multimedia transmission services, including the widest range of services such as VolP, videoconference, file transfer, streaming...This requires the coexistence of non real time traffic as well as delay sensitive flows with for example lower delay constraints but with tight data integrity targets and reciprocally. To be able to deal with the heterogeneous and various QoS requirements of multimedia services, the proposed FEC solution manages the QoE in a MAC/high-layers opportunistic approach. This relies on a generic approach of QoS management that implies that each traffic stream is considered as a service flow that has a specific QoS profile and possesses its own transmission buffer. Data integrity and delay which characterizes the QoS requirements of a service flow defined the QoS profile. Data integrity requirements are specified by $B E R_{\text {target, } k}$ for service flow $k$. Data integrity can be considered as guaranteed by the physical layer by adapting the modulation scheme and the transmit power to the mobile specific channel state. On the contrary, delay requirements are specified at the packet level and their achievements are directly impacted by the scheduler.

Clearly characterizing the delay requirements is necessary. We are convinced that the major constraint that can be considered in user communication is the limitation of the occurrences of large delay values. On this issue, the concept of delay outage can be applied. A delay outage is experienced by a packet of a given service flow $k$ when its delay is greater than its application specific threshold denoted $T_{k}$. The Packet Delay Outage Ratio $\left(P D O R_{k}\right)$ of each service flow $k$ is defined as the ratio of packets that do not meet the delay threshold constraint $T_{k}$ (out of delay) compared to the total number of packets transmitted. Fig. 2 shows an example of cumulative distribution of packet delay of a service flow $k$ at a given time instant. One of the objectives of the FEC scheduler is to regulate the experienced PDOR all along the lifetime of the service flow such as each PDOR value stays low and fairly distributed. This allows to ensure the satisfaction of the delay requirements at a short time scale as well as the service differentiation.

\subsection{Energy consumption minimization}

The last important goal of the FEC is to provide efficient energy radio resource allocation in addition to the system capacity and fairness increase. Current opportunistic resource mapping (as MaxSNR, PF or WFO for instance) basically overexploits multi-user diversity that usually leads to horizontal resource allocations. Indeed, since a flat fading is experienced during a frame on a given subcarrier [17], a same mobile often experiences the greatest channel condition on this one. This induces that classical opportunistic schedulers often allocate all the time slot of a subcarrier to a same mobile (which has to stay in active mode during a lengthy time). In addition, the probability to potentially have one different mobile selected on many available subcarrier is high. Consequently, during a same frame, many selected users can not be set in sleep mode for a low number of RUs allocated ${ }^{2}$. To conclude, although opportunistic resource allocation is widely acknowledged as the best way to manage wireless resources since they increase the system capacity and are potentially able to enhance the QoS, they often fail to be energy efficient.

Figure 3a gives conceptual MaxSNR resource allocation behavior. Its spectral efficiency provided is high but energy consumption also. On the contrary, Fig. 3b illustrates OEA resource allocation behavior. More vertical, its resource mapping allows to reduce energy consumption to the detriment of a spectral efficiency decrease (due to a lower multiuser diversity usage).

The FEC scheduler integrates a modified version of the energy efficient OEA solution [13], keeping its energy benefits while correcting its lacks in terms of system capacity and fairness. To achieve this goal, FEC extends the classical OEA opportunistic cross-layer approach to obtain a new vertical opportunistic resource mapping. When a user is in active mode, FEC tries, like OEA, to take benefit from its activation to compress its duration of activity and to transmit more data per "used" time slots. Thus, FEC scheduler highly increases sleeping mode duration and energy preservation. Originally, OEA scheduler used an "Energy Transmission Cost" $\left(E T C_{k}\right.$ ) parameter (in Watt). It is based on the energy cost of service flow $k$ to transmit on a RU:

\footnotetext{
2 Users consume a lot of power to transmit/receive few bit during a long time (with many allocated time slots but on few subcarriers).
} 


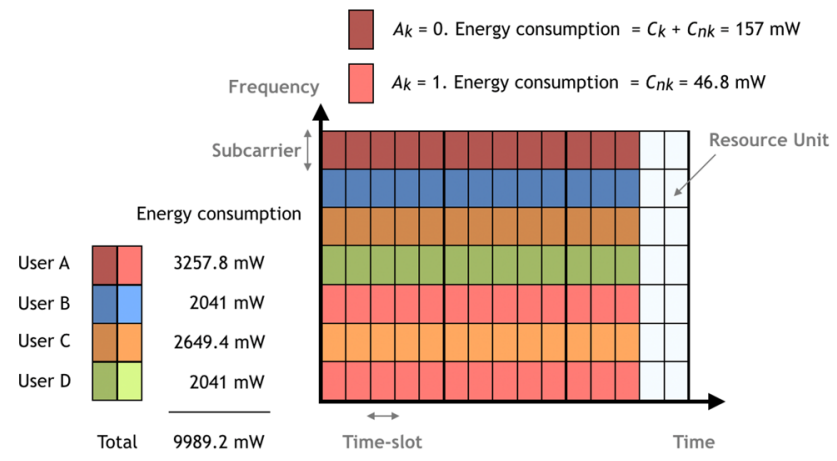

(a) Classical and horizontal opportunistic (i.e. MaxSNR) management of RUs.

Fig. 3 Conceptual scheduling strategies

$E T C_{k}=A_{k} * C n_{k}+\left(1-A_{k}\right) *\left(C_{k}+C n_{k}\right)$.

$A_{k}$ is fixed equal to 1 if the user of service flow $k$ is in active mode. Otherwise $A_{k}$ is set equal to 0 (i.e. sleep mode). $C n_{k}$ and $C_{k}$ are two constants (in Watt). $C_{k}$ is defined as the supplementary energy necessary to wake up the mobile $k$ from the sleep mode to the active mode (i.e. to supply energy to the radio module). $C n_{k}$ represents the energy required to transmit on a $n^{\text {th }}$ allocated subcarrier (i.e. energy required for the antenna transmission). The amount of energy required to be active (to switch the radio module on) is higher than the energy needed to transmit on an additional resource unit if the mobile is already set in active mode. Consequently, $C_{k}>C n_{k}$. In the following of this paper, $C_{k}$ and $C n_{k}$ values are considered respectively equal to $110.2 \mathrm{~mW}$ and $46.8 \mathrm{~mW}$, for all $k$ in accordance with measured hardware consumption [11].

The parameter $E T C_{k}$ is used in OEA scheduler and highly contribute on its energy efficiency. However, it has the negative side effect to widely limit the usage done of the multi-user diversity. This makes OEA unable to ensure optimized system capacity. In order to keep OEA energy minimization qualities while fixing this throughput and fairness issues, FEC integrates a modified $E T C_{k}$ parameter called "Context Aware Objectives Prioritization" parameter $C A O P_{k}$ :

$C A O P_{k}=A_{k} * C n_{k}+\left(1-A_{k}\right) *\left(\frac{C_{k}}{f\left(P D O R_{k}\right)}+C n_{k}\right)$,

where $f\left(P D O R_{k}\right)$ is:

$f\left(P D O R_{k}\right)=1+\beta * P^{\prime} D O R_{k}{ }^{\sigma}$.

The parameter $\sigma$ allows having the adequate sensitivity and reactivity to PDOR fluctuations in order to ensure high fairness at a short time scale. Extended studies have already been done to calibrate this function. A value of

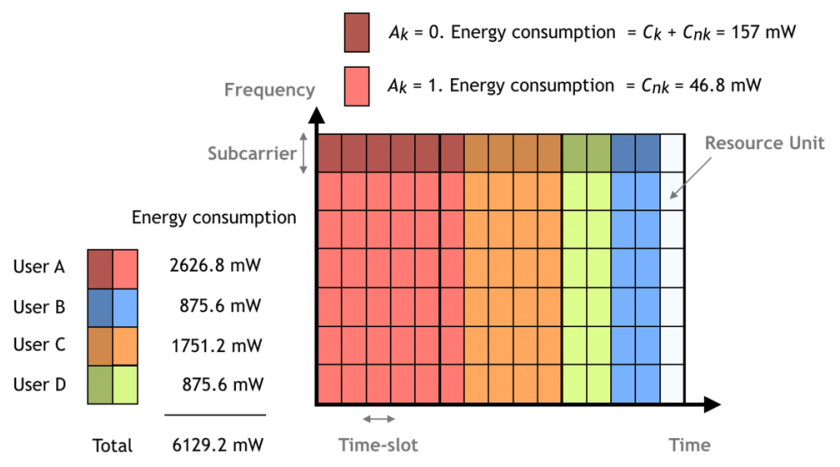

(b) Vertical Opportunistic Energy Aware (OEA) management of RUs.

$\sigma$ equal to 3 ensures the best trade-off between average performances and short term fairness and it is the value considered in the following [10]. In addition, $\beta$ is a normalization parameter. It ensures that $C A O P_{k}$ and $m_{k, n}$ are in the same order of magnitude. Given that $P D O R_{k}$ has an order of magnitude $10^{-2}, \beta$ is set to $10^{2 \sigma}$.

The higher $P D O R_{k}$ is, the more the system reduces $C A O P_{k}$ values and consequently the cost to activate a new mobile. The number of active users will increase, intensifying the multi-user usage and consequently making the spectral efficiency higher at the expense of the energy consumption (maximum $P D O R_{k}$ value $(100 \%)$ makes $C A O P_{k}$ constant and induces FEC similar to a MaxSNR resource allocation). At the opposite, low $P D O R_{k}$ values make FEC to decrease the number of active mobile in a same time. This results in an energy consumption reduction at the expense of the multi-user diversity usage. This provides a radio resource allocation relatively similar to OEA resource allocation algorithm (excepting that this version is strongly more fair due to Sect. refs3.2) .The contribution of this paper is to present a new scheduler that combines all previously described parameters that also uses the dynamic $P D O R_{k}$ parameter to adapt priority to QoS experienced by users and consequently to the context.

\subsection{FEC merging of priorities}

FEC algorithm is describe in Figs. 5 and 6. This scheduler allocates the radio resource $n$ to the service flow $k$ that have the greatest $F E C_{k, n}$ value such as:

$F E C_{k, n}=\frac{m_{k, n}}{A_{k} * C n_{k}+\left(1-A_{k}\right) *\left(\frac{C_{k}}{f\left(P D O R_{k}\right)}+C n_{k}\right)}$.

As shown in Fig. 4, the probability for a mobile to receive $\mathrm{RUs}$ is highly relied on the magnitude of its $F E C_{k, n}$. Considering $m_{k, n}$ allows to increase system capacity avoiding unprofitable radio resource allocation. Thanks to 


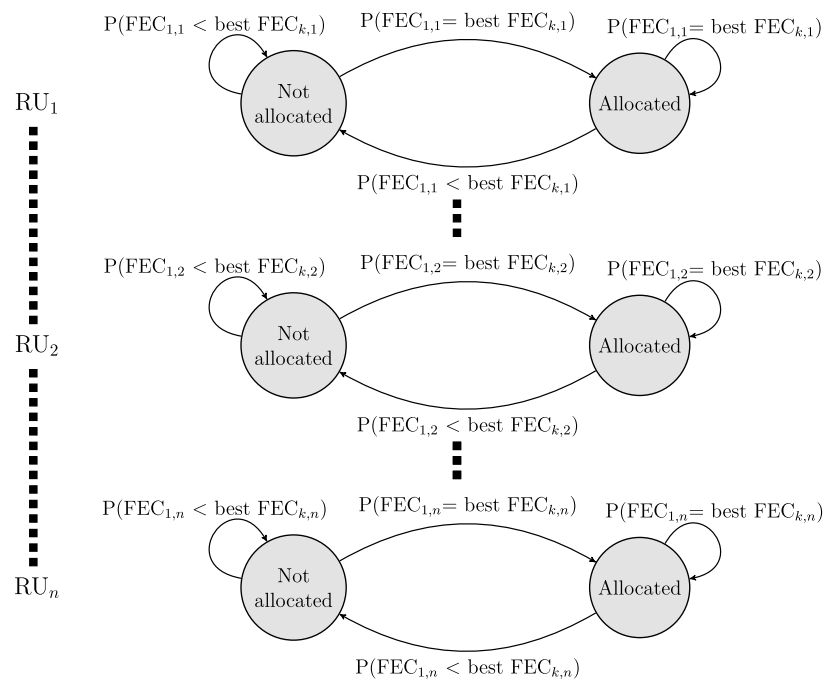

Fig. 4 Allocation probabilities Markov chain for mobile 1 with FEC scheduler

$f\left(P D O R_{k}\right)$ function, FEC also ensures service differentiation: if a mobile experienced QoS decrease due to traffic burst, hard delay constraints or high BER requirements, it has proportionally more chance to be activated in the scheduling process with FEC. By adjusting the multi-user diversity usage thanks to a good function of $f\left(P D O R_{k}\right)$, FEC adequately adjust the number of activated users per timeslot to the minimum possible in order to always make efficient energy management while respecting the QoS requirements. If the system experiences difficulties, FEC increases the multi-user diversity thanks to its ability to consider the raised values of $P D O R_{k}$ which urging it to obtain a better spectral efficiency. This avoids users QoS decrease while guaranteeing a relative fairness and service differentiation. This also adequately improves the multiuser usage of the scheduler and consequently the system capacity when required by the load context or by higher layer user application constraints. The other parameters allows to fight versus energy waste.

In the following, a possible version of the proposed scheduling algorithm is described step by step:

- Step $1=>$ The scheduler updates the current $P D O R_{k}$ and $B O_{k}$ values of each service flow $k$ and estimate the $m_{k, n}$. Then $t$ and $n$ are initialized to 1 , then $A_{k}$ to 0 (user of the service flow $k$ is in sleep mode). Afterwards, $C A O P_{k}$ and $F E C_{k, n}$ are computed for each service flow and each subcarrier.

- Step $2=>$ For subcarrier $n$, the FEC scheduler selects the service flow $k$ with the greatest $F E C_{k, n}$ value.

- Substep $2.1=>$ If the buffer occupancy of service flow $k$ is positive, the scheduler goes to Substep 2.2. Else, if all buffers are empty, the FEC scheduler goes to Step

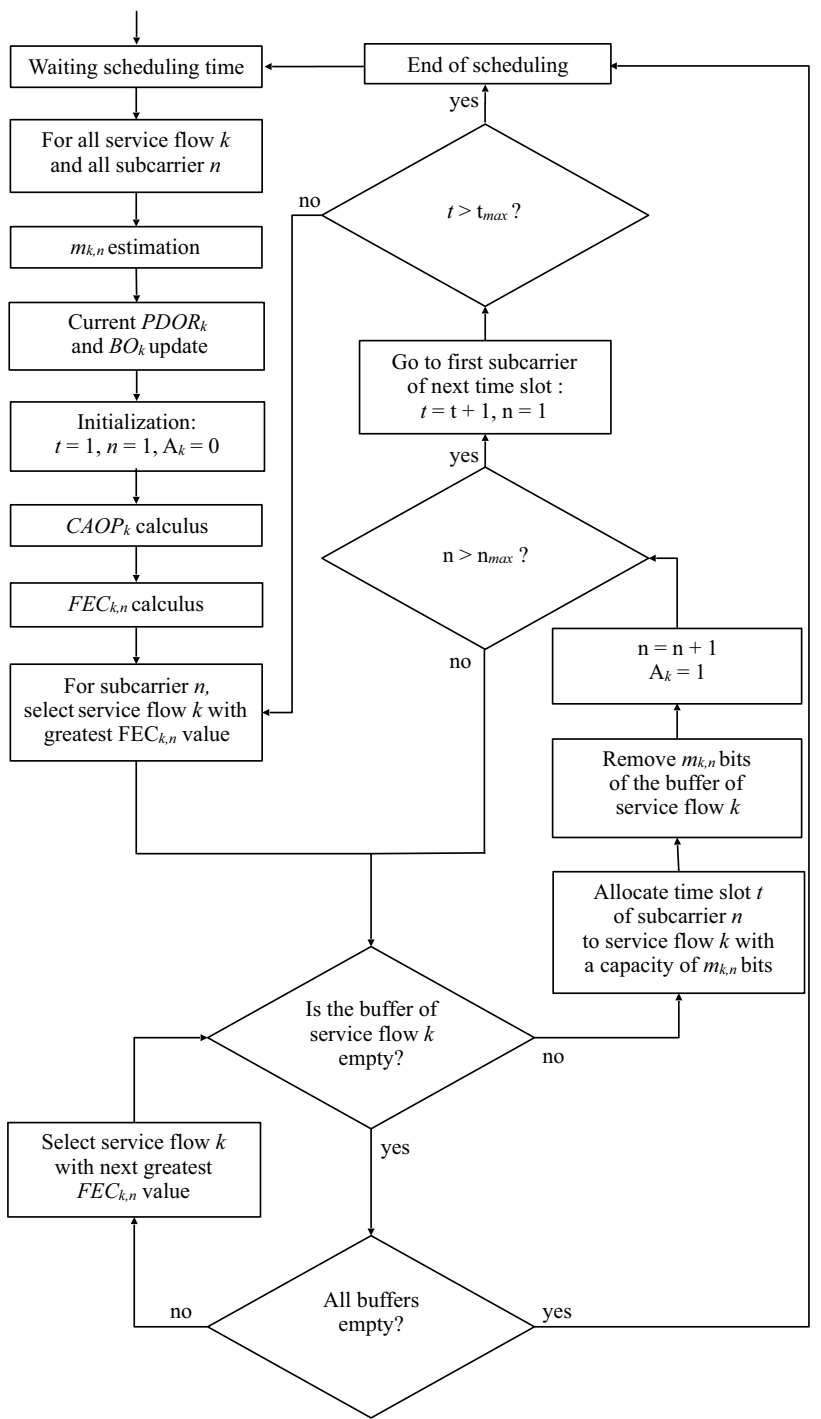

Fig. 5 FEC's flow chart

3 , otherwise, it selects the next service flow $k$ with the greatest $F E C_{k, n}$ value and restarts Substep 2.1.

- Substep $2.2=>$ The scheduler allocate time slot $t$ of subcarrier $n$ to service flow $k$ with a capacity of $m_{k, n}$ bits. Then it removes $m_{k, n}$ bits of the buffer of service flow $k$, increments the value of $n$ and set $A_{k}$ to 1 (user of service flow $k$ in active mode). If the value of $n$ is smaller than the maximum number $n_{m}$ ax of subcarriers, the scheduler goes to Substep 2.1 for allocating the next subcarrier. Else, it goes to the next substep.

- Substep $2.3=>$ Increment the value of $t$ and set $n$ to 1 . If $t$ is smaller than the maximum number $t_{k, n}$ of time slots by subcarrier, go to Step 2 for allocating the subcarriers of the next time slot. Otherwise, go to Step 3. 


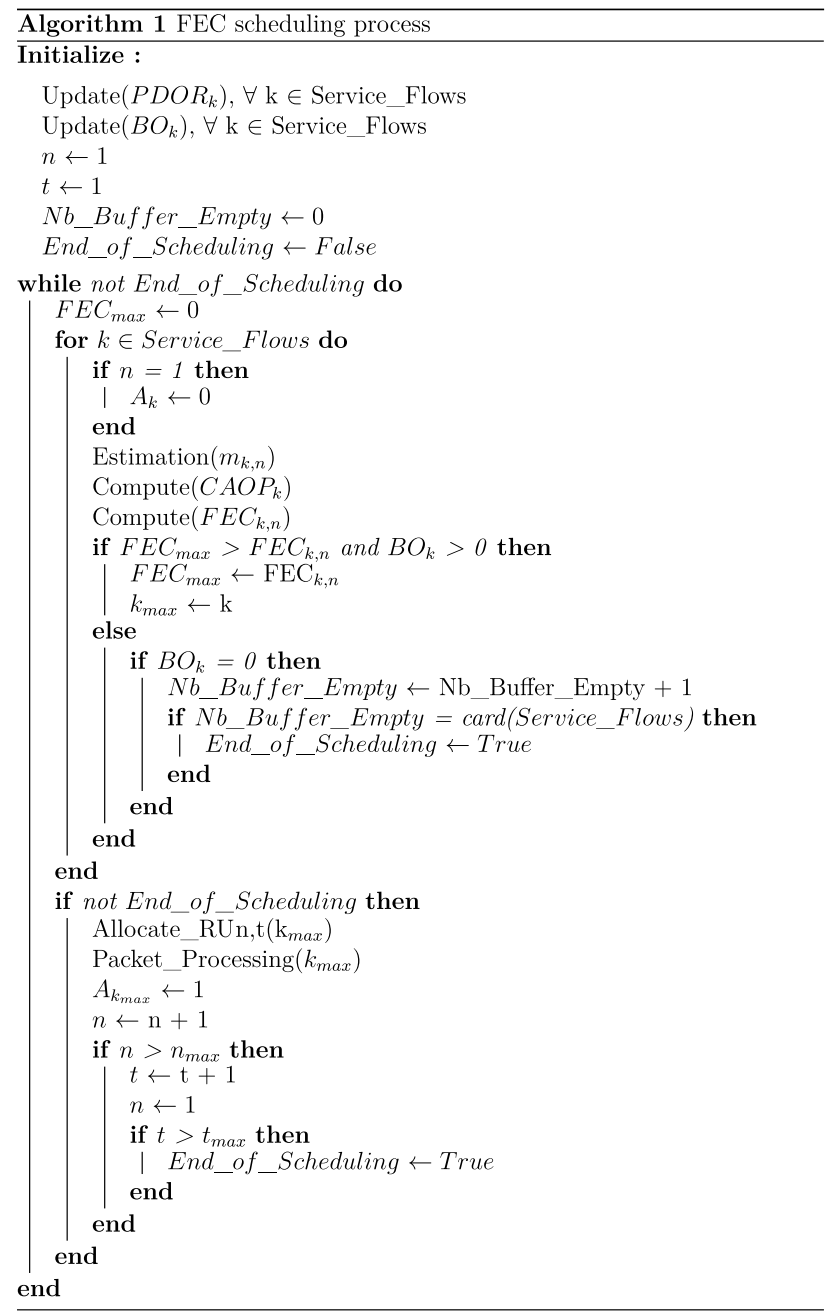

Fig. 6 FEC's pseudocode

- Step $3=>$ All buffers of each service flow $k$ are empty or all subcarriers of all time slots are allocated : the scheduling ends.

\subsection{FEC overhead and complexity discussion}

FEC scheduler needs some inputs to work such as:

- User buffer occupancy (as all centralized schedulers),

- User SNR (like MaxSNR, OEA, PF WFO and DT solution),

- User PDOR (like WFO),

- User activity $A_{k}$ (like OEA and DT).

Since buffer occupancy and SNR are used by all efficient solutions (MaxSNR, WFO, PF, DT...), FEC scheduler has no complexity addition on these points. PDOR is a simple integer to forward regularly to the access point but this can be done at a larger scale than the scheduling time. Collecting this input is consequently feasible and doesn't provide significant overhead. $A_{k}$ is directly determined by the scheduler in its decision process and occurs no overhead. Considering the algorithm complexity, FEC scheduling operates sorting of $F E C_{k, n}$ for each user $k$ for all subcarriers $n$ as MaxSNR makes with $S N R_{k, n}$. Consequently, the proposed FEC algorithm can be considered to have a close complexity than MaxSNR. The only supplementary complexity is the computation of each $F E C_{k, n}$ that require 2 divisions, 2 multiplications and 2 addition more than with MaxSNR but with integers (and sometime just with 0 and 1) that can be considered widely acceptable.

\section{Performance evaluation}

In this section, the proposed Fair Energy efficient with high system Capacity scheduler (FEC) is compared to 5 schedulers. They are well known in the literature and/or highly efficient in at least one objective that is in the scope of action of our proposed solution (i.e. spectral efficiency, energy consumption and QoS).

Round Robin (RR) this simple scheme allows to compare the proposed solution with a classical state of the art nonopportunistic scheduler [3].

Maximum Signal to Noise Ratio (MaxSNR) this opportunistic scheduler is widely acknowledged to its ability to widely increase the capacity of wireless networks [4].

Weighted Fair Opportunistic (WFO) this solution is able to ensure high fairness and service differentiation. It also outperforms other well-known fair opportunistic schedulers like Proportional Fair (i.e. [10]).

Opportunistic Energy Aware (OEA) this user selection strategy is focused on energy consumption minimization [13]. Contrary to T-MAC solution, it keeps some benefits of the multi-user diversity usage and represents a more challenging point of comparison (these solutions are compared in [11]).

Dynamic Trade-off (DT) this scheduler has the ability to switch of behavior depending of the traffic load context and leads to a very efficient trade-off between energy consumption and spectral efficiency [11].

Discrete event simulations are used to obtain performance evaluation. The global simulation setup is given in Table 1:

It is also considered that all users run realistic Variable Bit Rate applications that generates high volume of data with important sporadic and tight delay requirements which significantly complicates the task of the resource allocation algorithm. Each mobile has a traffic composed of an video and voice streams [19-21] but with different required throughputs and/or delay constraints according to the considered scenario. 
Table 1 Global simulation setup

\begin{tabular}{ll}
\hline Parameters & Values \\
\hline Number of subcarriers $n_{\text {max }}$ & 32 \\
Number of time slots $t_{\text {max }}$ & 10 \\
Wake up cost $C_{k}$ & $110.2 \mathrm{~mW}$ \\
Transmission cost $C_{k}$ & $46.8 \mathrm{~mW}$ \\
Target BER & $10^{-3}$ \\
Average $\alpha_{k, n}$ value & 1 \\
Subcarrier spacing & $15 \mathrm{kHz}$ \\
Cell radius & $500 \mathrm{~m}$ \\
Simulation duration & $5.10^{5}$ frames \\
\hline
\end{tabular}

Table 2 First scenario setup

\begin{tabular}{llll}
\hline Group & Distance $\left(d_{k}\right)$ & $\begin{array}{l}\text { Delay threshold } \\
\left(T_{k}\right)\end{array}$ & Data rate $\left(D_{k}\right)$ \\
\hline 1 & $100 \mathrm{~m}$ & $80 \mathrm{~ms}$ & $150 \mathrm{Kbps}$ \\
2 & $200 \mathrm{~m}$ & $80 \mathrm{~ms}$ & $150 \mathrm{Kbps}$ \\
\hline
\end{tabular}

Four simulation scenarii are shown in the presented performance evaluation. First, the behavior of the schedulers when mobiles occupy different geographical positions are analyzed. The second scenario underlines the performance of the ressource allocation algorithms when mobiles have heterogeneous bit rate requirements. In the third scenario, QoS differentiation concerning applications with different delay requirements is evaluated. The fourth simulation scenario gather the previous ones and considers mobiles with all three: heterogeneous geographical positions, bit rate and delay requirements.

\subsection{Scenario 1: distance influence study on the schedulers performances.}

It is widely acknowledged that, in wireless networks context, the mobiles close to the access point usually obtain better QoS than mobiles more distant due to their higher mean spectral efficiency. In this scenario, a first half of mobiles is situated close to the access point and the second half is twice farther from the access point to study the impact of heterogeneous distance on the scheduling performances (fairness behavior, capacity to optimize the usage of the multi-user diversity to increase system capacity, etc.). The other parameters are fixed for all the mobiles and described in Table 2. In addition, performance evaluations, on each criterion, are carried out studying the influence of the traffic load (underlining the different scheduler behaviors in low traffic load and high traffic load contexts). The network traffic load increases in our simulations by adding users 2 by 2 (each time, 1 close user and 1 far user).
Spectral efficiency and throughput

Figure 7a shows, for each scheduler, the spectral efficiency obtained with different traffic load in the system. Since RR is not opportunistic, it does not take any benefit of multi-user diversity and its spectral efficiency is constant $^{3}$ and low. Energy focused scheduler (OEA), significantly limit the multi-user diversity usage in their allocation process offering lightly better results. At the opposite, MaxSNR is highly opportunist and grants an important spectral efficiency gain. However, as explained in Sect. 3.1, MaxSNR has a severe lack on fairness and is not able to take all the advantages of the multi-user diversity and is slightly outperformed in highly loaded context by WFO and DT that are fairer ${ }^{4}$.

Thanks to its dynamic CAOP parameter based on the PDOR values, FEC has a lesser spectral efficiency in low traffic load context using a moderate usage of the multiuser diversity concentrating its efforts on energy (Fig. 7a). Nonetheless, when it appears necessary [when energy focused schedulers approach congestion (Fig. 7b)], its CAOP factor adequately increases. This induces FEC raises its usage of the multiuser diversity which improves the spectral efficiency at the same level than MaxSNR (allowing to reach the same high overall system capacity limit [Fig. 7b)].

\subsubsection{Energy consumption}

The abilities of each scheduler to be more or less energy efficient can be studied thanks to the analysis of Fig. 7c. Clearly, the Round Robin scheduler (RR) is the solution that provides the worst results. With RR that is not opportunistic, a non negligible amount of $\mathrm{RU}$ are allocated with a poor spectral efficiency rentability (i.e. in term of bit per $\mathrm{RU}$ ). This results in high energy and RU wastes. In addition, RR allocation patern is regular and cyclic. Consequently, with RR, many users are simultaneously activated and, as explained in Sect. 3.3, this exacerbates the energy waste since more mobiles have to pay the high radio transmission activation price $C_{k}$. Focusing on the RR curves, we can notice that with more than 20 mobiles, the RR curve increases more slowly. The explanation is that after this

\footnotetext{
3 When system congestion occurs, RR spectral efficiency slightly increase due to the fact that close mobiles pick up the $\mathrm{RU}$ previously done to far users in order to achieved an equal ratio of $50 \%$ assigned to each group.

${ }^{4}$ However when WFO reach its provided system capacity limit (24 users), it tries in vain to help far users (increasing their priority) which reduce its ability to take full benefit of the multiuser diversity usage. In addition, in highly overloaded system (more than 30 users), all user's PDOR are equal to 1 and WFO scheduling becomes similar to MaxSNR which explain a same spectral efficiency)
} 


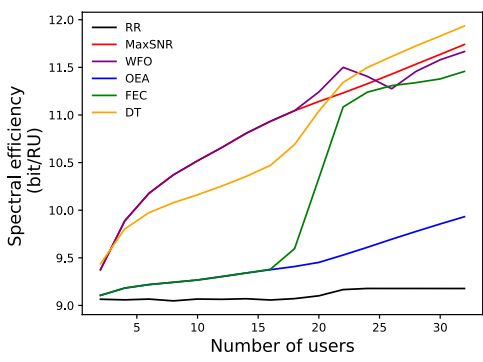

(a) Spectral efficiency.

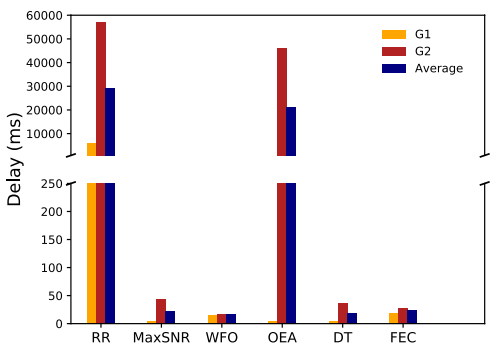

(d) Mean packet delay for a traffic load of 22 users.

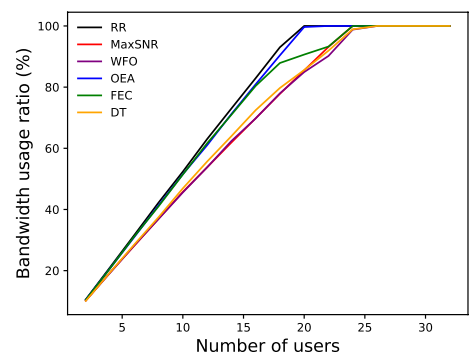

(b) Bandwidth usage ratio.

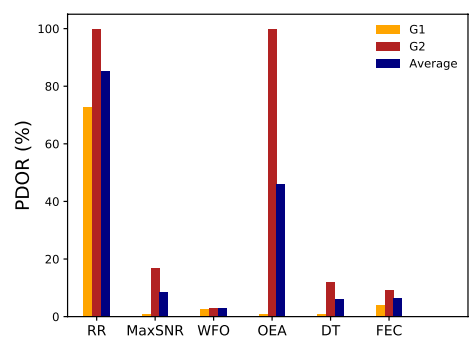

(e) Mean PDOR values for a traffic load of 22 users.

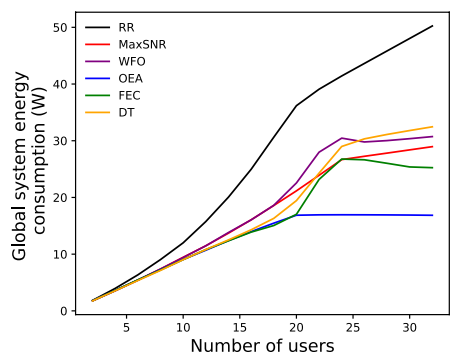

(c) Energy consumption.

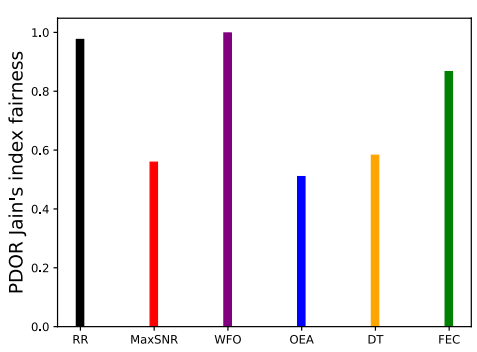

(f) Scheduler Jain index fairness (traffic load of 22 users).

Fig. 7 Scenario 1 (distance influence on the schedulers performances)

threshold, the cell is overloaded. RR does not success to provide enough RUs to each user to reach their application requirements. Due to the lack of radio resource unit, the users are more often obliged to wait a long time in sleep mode even with data to transmit. Consequently, each user consumes in overall less energy over the time after the system congestion threshold is crossed.

Another approach is OEA resource allocation strategy. Its concept is to drastically limit the usage of the multiuser diversity to a low value. This induces that this solution ensures low energy consumption whatever the traffic load considered. However, this result must be put into perspective since OEA keeps to focus on energy consumption minimization even when traffic loads is high. This stubborn radio resource management leads OEA solution to quickly reach congestion (Fig. $7 \mathrm{~b}$ ) and to provide high delay occurrences (Fig. 7d).

Contrary to OEA, WFO fully exploits the multiuser diversity thanks to its extended opportunistic approach and extends the system capacity (Fig. 7b). Not focusing on energy consumption, WFO is outperformed on this criterion by OEA. However, it outperforms RR (Fig. 7c) thanks to a strongly better spectral efficiency (Fig. 7a). About WFO performance, it is important to notice that when the system is overloaded [after 24 users (Fig. 7b)], WFO unfruitfully tries to ensure fairness helping far users (increasing their priority). Activated less often close mobiles and more the far mobiles, the number of activated mobile is reduced and consequently more mobiles are set in sleep mode which stabilize WFO energy consumption.

Regarding MaxSNR, we can notice that MaxSNR has slightly better energy results than WFO and particularly when system is overloaded. Indeed, when the system is overloaded, this scheduler has a propensity to segregate a part of users (far from the access point) and consequently it obtains reduced benefits of multiuser diversity usage. This is a weakness in term of spectral efficiency and fairness but an advantage to increase user sleep duration and reduce energy consumption.

Considering underloaded contexts (i.e. number of users inferior to 18 in this scenario), due to large excess of available radio resource units (Fig. 7b), guaranteeing high QoS is easily achievable by DT and FEC. Thanks to their system of weights that dynamically adjust the objectives priority to the context, they consequently focus their priorities on energy (Fig. 7c) rather than system throughput (Fig. 7a). However, considering the network traffic load, DT does not fully optimizes the trade-off. FEC, thanks to its CAOP parameter based on QoE measured is more accurate. It outperforms DT and offers energy gain equal to OEA until this stay possible.

Considering highly loaded context (number of users between 18 and 24), the lack of available RUs (Fig. 7b) requires schedulers to focus more on system capacity than energy consumption which then becomes a lower priority in order to preserve QoS. In this context, DT and FEC 
decide to slightly sacrifice energy in order to sustain the network viability and then favor high spectral efficiency proportionally to the traffic load with DT and proportionally to users QoE difficulties with FEC. However, performing the energy-throughput transition according to the evolution of the traffic load is not accurate and leads the DT to make its transition too early in this scenario. Thanks to is CAOP parameter based on PDOR, FEC begins its energythroughput transition just when difficulties really occur and provides greater and longer energy gain (Fig. 7c).

In overloaded context (number of users superior at 24), since all user experienced high delay and consequently high PDOR, FEC scheduling becomes close to MaxSNR which is close to be the best strategy in this specific case. Consequently, these schedulers offer slightly the same results in term of spectral efficiency (Fig. 7a), system capacity (Fig. 7b) and energy (Fig. 7c).

\subsubsection{Delay and fairness}

An important Quality of Service Key Performance Indicator (KPI) is the packet latency. Figure $7 \mathrm{~d}$ shows the mean packet delay in the system in milliseconds and Fig. 7e the mean PDOR when system load is high (26 users) which potentially makes users experience some difficulties. Note that 2 groups emerged:

- First, RR and OEA that have the worst results since they quickly experienced congestion. Since they had a low spectral efficiency (Fig. 7a), they do not success to support a significant amount of traffic load with acceptable QoS.

- Secondary, MaxSNR, WFO, DT and FEC (each one being opportunitic algorithm solution) are able to better endure higher load increase with satisfactory delay and PDOR.

Concerning Fairness about distance, results show (Fig. 7d-f) that RR, OEA, MaxSNR and DT significantly penalize user far from the access point (group 2). OEA and MaxSNR are the most unfair due to their pure opportunistic approach that blindly favors users with good SNR. RR and DT ensure a same amout of RUs that slightly improve this behaviors without completely solving the problem. Indeed, giving users the same amount of RUs does not mean to allow them to transmit the same amount of bits as it depends on their position (due to the path loss attenuation on SNR). WFO is conceived in order to ensure high fairness and consequently offer the best results on this metric. However, the proposed FEC scheduler is close to WFO results (between WFO and DT) ensuring good fairness (Fig. 7f) with low delay and PDOR values for all groups
Table 3 Second scenario setup

\begin{tabular}{llll}
\hline Group & Distance $\left(d_{k}\right)$ & $\begin{array}{l}\text { Delay threshold } \\
\left(T_{k}\right)\end{array}$ & Data rate $\left(D_{k}\right)$ \\
\hline 1 & $100 \mathrm{~m}$ & $250 \mathrm{~ms}$ & $300 \mathrm{Kbps}$ \\
2 & $100 \mathrm{~m}$ & $250 \mathrm{~ms}$ & $100 \mathrm{Kbps}$ \\
\hline
\end{tabular}

as well as high system capacity. However, this is achieved by the FEC with, in addition, high energy gain.

\subsection{Scenario 2: heterogeneous bit rate sources impact on scheduler performances}

Here, users are divided in two groups that differ only by their data rate requirements as described in Table 3. This allows to study the ability of each scheduler to manage mobiles using applications with various data rate. Results are shown in Fig. 8.

As expected, RR provides the worst performances due to its non opportunistic RUs management. Many energy is wasted, system capacity limit is low and users that require high throughput fail to meet their delay requirements. Limiting the usage of multiuser diversity, OEA offers large energy gain but provides poor system capacity (slightly superior than with RR), QoS and fairness. On the contrary, with MaxSNR, spectral efficiency is increased at the expense of energy and fairness. WFO, which is able to ensure service differentiation, successfully corrects this lack of fairness while staying close to MaxSNR other performances (on spectral efficiency ${ }^{5}$ and system capacity). Thanks to their abilities to adjust the objectives priority according to the context, DT and FEC provide low energy consumption in underloaded context (Fig. 8c) since no difficulties are met by users. When traffic load increases, they make their energy-throughput transition in order to raise the system capacity (Fig. 8a) and hold QoS requirement ${ }^{6}$. However, since DT is unable to ensure service differentiation, it fails to guarantee high fairness and users of group 1 experience difficulties at the opposite of FEC that success to ensure an high fairness (Fig. 8f) and high QoS ( (Fig. 8d and PDOR28) like with WFO for all groups.

\footnotetext{
5 in overloaded context WFO spectral efficiency decreases since mobiles with high throughput requirements experience huge difficulties giving them disproportionate priority. This makes WFO using a truncated multiuser diversity. This decreases its spectral efficiency but also its global energy consumption.

${ }^{6}$ In overloaded context DT offers the same spectral efficiency than MaxSNR since, in this scenario, all users are at the same distance from the access point and no supplementary of multiuser diversity can be used by DT compared to MaxSNR (contrary to the scenario 1).
} 


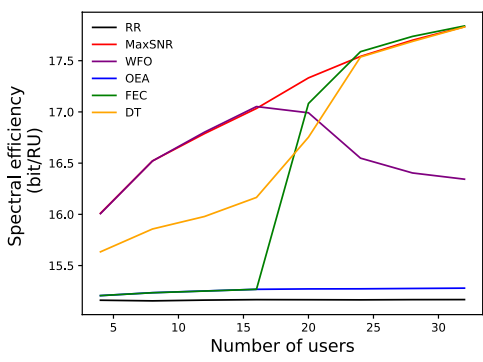

(a) Spectral efficiency.

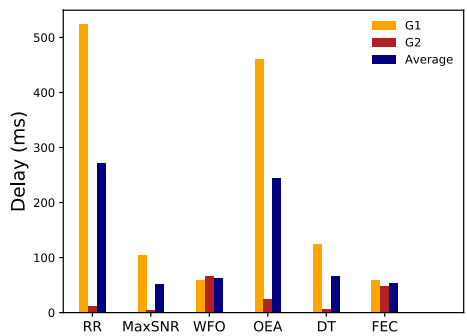

(d) Mean packet delay for a traffic load of 24 users.

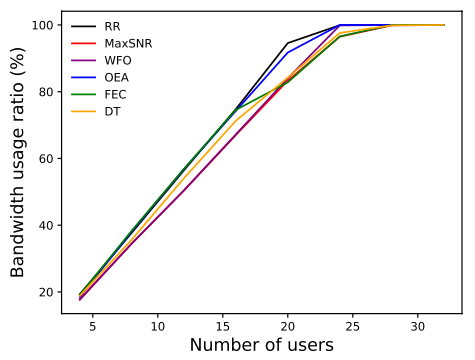

(b) Bandwidth usage ratio.

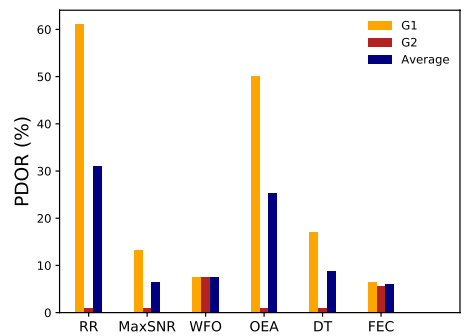

(e) Mean PDOR values for a traffic load of 24 users.

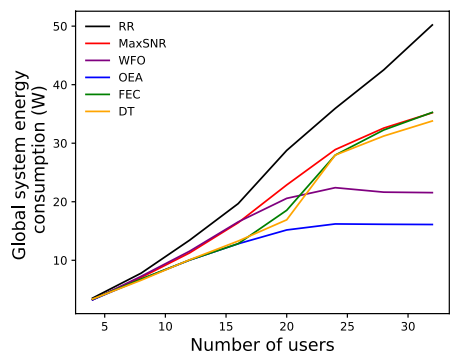

(c) Energy consumption.

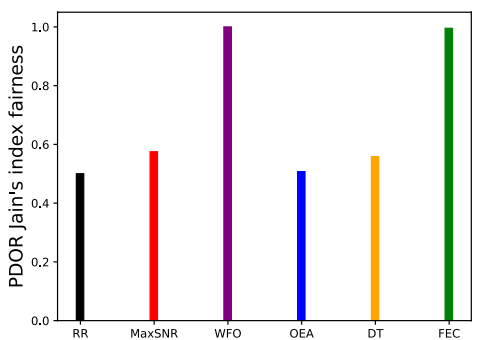

(f) Scheduler Jain index fairness (traffic load of 24 users).

Fig. 8 Scenario 2 (performance with heterogeneous bit rate sources)

\subsection{Scenario 3: heterogeneous delay constraints impact on scheduler performances}

In this scenario the influence of heterogeneous delay requirements on the scheduling performances is studied. In this simulation scenario, users are split into two groups that differ only by their delay requirements (cf. Table 4).

Results are shown Fig. 9 and underline the same tendency for each schedulers than in scenario 2. However, they allow some new analysis/discussions:

- The DT triggers its "energy-throughput" transition based on system capacity traffic load measures. This information, although interesting, is not accurate and makes DT to initiate its transition too late when users experienced difficulties since a while (contrary to the scenario 1 where it was too early). Consequently, in this scenario and like OEA scheduler, DT offers better energy consumption than FEC (Fig. 9c) but at the expense of user satisfaction (Fig. 9e). In addition, FEC offers strong service differentiation close to WFO results and widely outperforms DT offering a same PDOR level for all group whatever their delay constraints (Fig. 9e, f).

- This scenario underlines that many un-equivalent level of fairness can be offered by a scheduler to its users. The basic approach is to consider as fair an allocation of a same number of RUs to each users (like with RR) but this does not guarantee a same throughput and is far to be a relevant definition. More evolved approaches consider fair to offer a same packet delay to all users. However, the greatest level of fairness can be considered as to ensure to all mobiles a same degree of satisfaction whatever their distances, application throughput requirements or delay constraints. Consequently, in this scenario, ensuring high fairness between mobile with different delay requirement is not equal to ensure to all users a same packet delay like provided by MaxSNR and DT (Fig. 9d). In this scenario, the objectives are to guarantee a packet delay for the user of group 1 inferior to $50 \mathrm{~ms}$ and for users of group 2 inferior to $250 \mathrm{~ms}$. Fairness can be adequalty measured as providing a same ratio of packets in delay outage (i.e. a same PDOR) for all groups. Following this analysis, only WFO and FEC schedulers are able to offer a good fairness in this scenario (Fig. 9e, f).

\subsection{Scenario 4: global scheduling performances analysis}

Previously, the behavior of each scheduler has been studied in simple contexts considering one criterion at a time to better understanding their influences on the performances. In order to compile these ones, this section shows a study of the performance of the evaluated protocols in a 


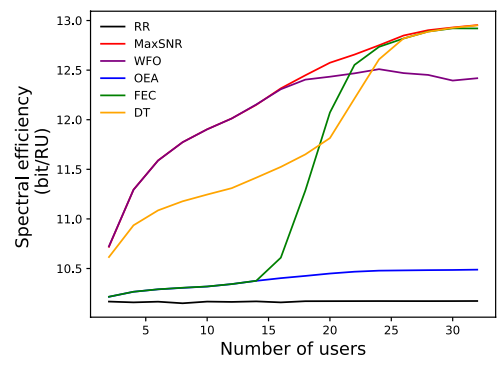

(a) Spectral efficiency.

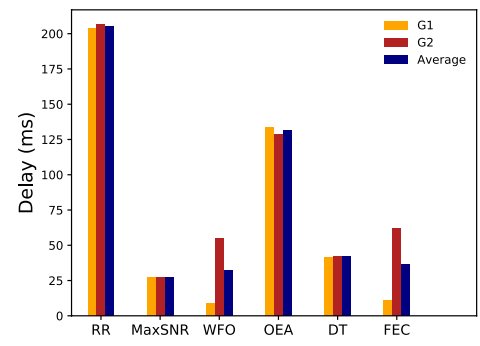

(d) Mean packet delay for a traffic load (e) of 20 users.

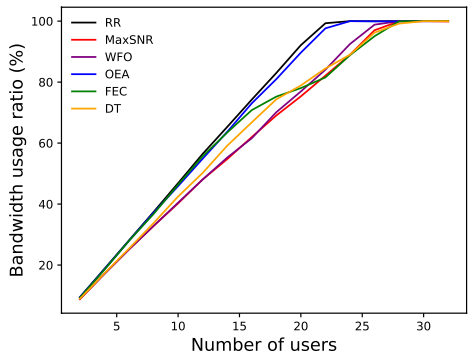

(b) Bandwidth usage ratio.

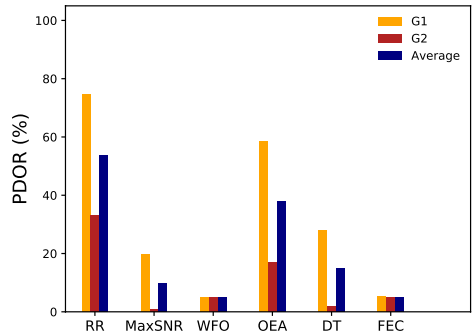

(e) Mean PDOR values for a traffic load (f) of 20 users.

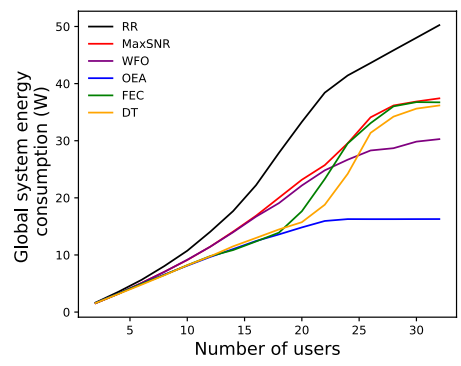

(c) Energy consumption.

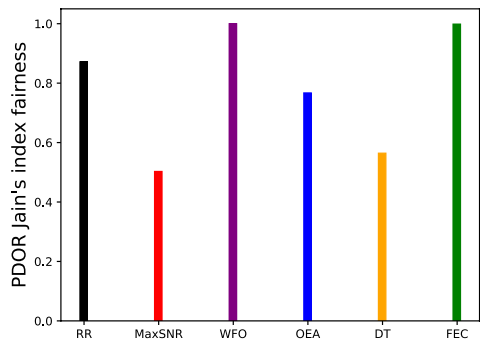

Fig. 9 Scenario 3 (performance with heterogeneous delay constraints)

Table 4 Third scenario setup

\begin{tabular}{llll}
\hline Group & Distance $\left(d_{k}\right)$ & $\begin{array}{l}\text { Delay threshold } \\
\left(T_{k}\right)\end{array}$ & Data rate $\left(D_{k}\right)$ \\
\hline 1 & $100 \mathrm{~m}$ & $50 \mathrm{~ms}$ & $150 \mathrm{Kbps}$ \\
2 & $100 \mathrm{~m}$ & $250 \mathrm{~ms}$ & $150 \mathrm{Kbps}$ \\
\hline
\end{tabular}

Table 5 Fourth scenario setup

\begin{tabular}{llll}
\hline Group & Distance $\left(d_{k}\right)$ & $\begin{array}{l}\text { Delay threshold } \\
\left(T_{k}\right)\end{array}$ & Data rate $\left(D_{k}\right)$ \\
\hline 1 & $50 \mathrm{~m}$ & $250 \mathrm{~ms}$ & $100 \mathrm{Kbps}$ \\
2 & $100 \mathrm{~m}$ & $250 \mathrm{~ms}$ & $100 \mathrm{Kbps}$ \\
3 & $50 \mathrm{~m}$ & $250 \mathrm{~ms}$ & $300 \mathrm{Kbps}$ \\
4 & $100 \mathrm{~m}$ & $250 \mathrm{~ms}$ & $300 \mathrm{Kbps}$ \\
5 & $50 \mathrm{~m}$ & $80 \mathrm{~ms}$ & $300 \mathrm{Kbps}$ \\
6 & $100 \mathrm{~m}$ & $80 \mathrm{~ms}$ & $300 \mathrm{Kbps}$ \\
7 & $50 \mathrm{~m}$ & $80 \mathrm{~ms}$ & $100 \mathrm{Kbps}$ \\
8 & $100 \mathrm{~m}$ & $80 \mathrm{~ms}$ & $100 \mathrm{Kbps}$ \\
\hline
\end{tabular}

more general context. 8 heterogeneous groups of mobiles are considered here as described in Table 5.

Figure 10a shows the global energy consumed with each scheduler when traffic load represents around $50 \%$ of the system capacity limit. As expected, RR is the most energy greedy since this resource allocation provides inefficient spectral efficiency and horizontal resource allocation which induces users are activated on many time slot to transmit few bits. MaxSNR and WFO offer better results due to their opportunistic users-RUs managements which provide better spectral efficiency. However, they are respectively widely outperformed by DT, FEC and OEA that try to have a more vertical resource allocation which highly compress user time activity and consequently significantly increase user time sleep duration.

Figure 10b, c respectively show the spectral efficiency and bandwidth usage ratio for each scheduler in high traffic load context. RR provides, again, poor results since it does not take into account the wireless transmission conditions in its resource allocation process. Too many focused on energy objective, OEA fails to ensure significant improvement on these metrics. At the opposite, MaxSNR and WFO that overexploit the multiuser diversity provide huge benefits: high spectral efficiency (Fig. 10b) and low bandwidth usage ratio [i.e. high system capacity limit (Fig. 10c)]. DT and FEC are doing their "energy - system capacity" transition in order to support the traffic load growth and reach close results to MaxSNR.

Figure 10d-f highlight the inabilities of RR, MaxSNR, OEA and DT to provide service differentiation. When delay requirements are stringent and/or the path loss is high and/or the source bit rate is raised, mobiles experienced 


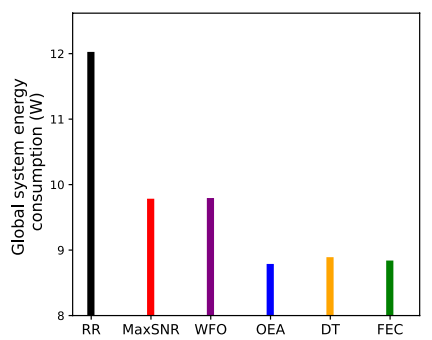

(a) Energy consumption for a traffic load of 16 users.

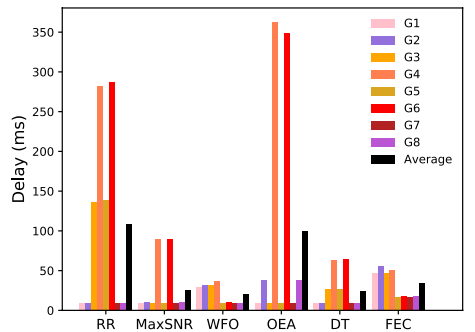

(d) Mean packet delay for a traffic load (e) of 32 users.
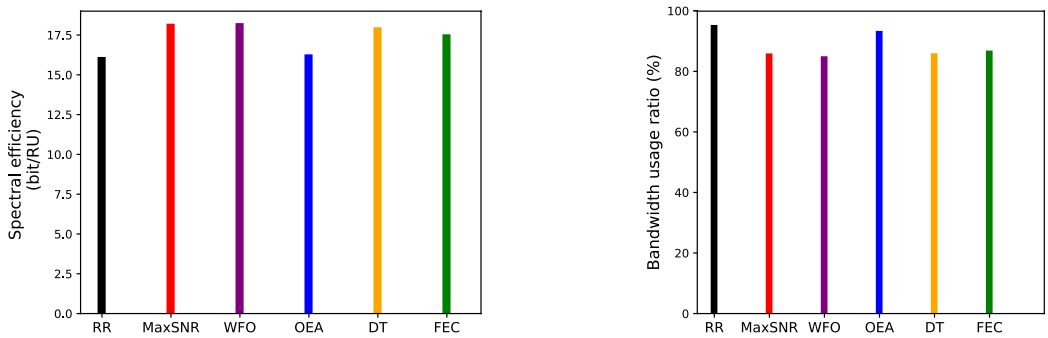

(b) Spectral efficiency for a traffic load of 32 users.

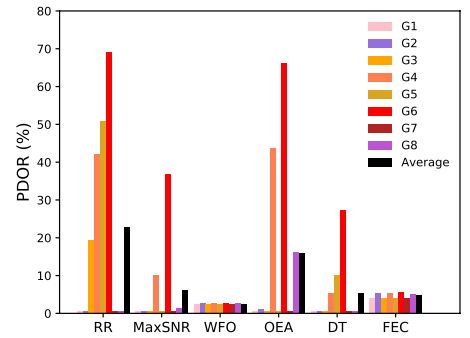

(e) Mean PDOR values for a traffic load of 32 users. (c) Bandwidth usage ratio for a traffic load of 32 users.

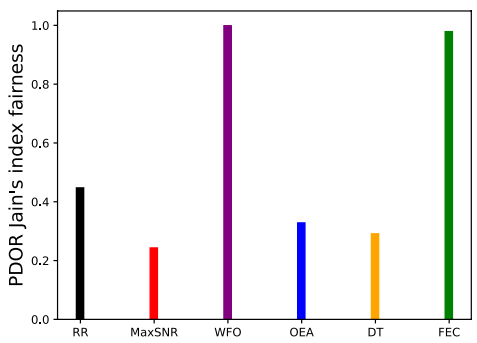

(f) Scheduler Jain index fairness (traffic load of 32 users).

Fig. 10 Scenario 4 (global scheduling performances analysis)

severe difficulties (particularly the group 6). On the contrary, WFO and FEC success to differentiate users according to their profile and position. They guarantee to each group the respect of their heterogeneous delay requirements (Fig. 10d), withdrawing negative impact of distance (Group 1, 3, 5, 7 are no more penalized) and consequently offer a same amount of PDOR/QoE to each group (Fig. 10e) as well as a good fairness (Fig. 10f).

\section{Conclusion}

Jointly guaranteeing high system capacity, high fairness, high QoE and low system energy consumption is very challenging in wireless networks. Specialized solutions as MaxSNR, WFO or OEA have been well designed to meet one of these criteria but by failing others. Alternative works (like DT) rely on the usage of tradeoffs but provide un-optimized/averaged performance. This paper proposes a new paradigm: the magnitude of importance of these objectives could vary according to the context. In very low traffic load context, radio resource units are abundant and energy minimization should be the main objective. With the rise of the network traffic load, more focus/priority should be done on spectral efficiency and system capacity increase in an adequate "energythroughput" management. In high traffic load, the spectral efficiency increase should become the primary objective in order to continue to satisfy the maximum number of users and energy minimization priority must be relegate. In this paper, a new access scheme called FEC scheduler has been proposed. It allows to tune users' priorities and the multiuser usage benefit according to users' requirements and networks difficulties. Performance evaluation shows that the proposed solution provides together a level of fairness, energy efficiency and system capacity close to the acknowledged specialized schedulers on these 3 major performance indicators: without penalizing the system capacity (reaching almost the same spectral efficiency than MaxSNR), it provides efficient global system energy consumption (very close to OEA energy specialized scheduler) while ensuring high level of user satisfaction, QoS differentiation and fairness (close to WFO fair specialized scheduler). This results in a scheduler that widely outperforms existing solution trying to ensure these $3 \mathrm{KPIs}$ together as DT solution. In future works, a study of energy efficient multi-cells schedulers will be done.

\section{Compliance with ethical standards}

Conflict of interest The authors declare that they have no conflict of interests. 


\section{References}

1. Simon C, Leus G (2009), Round-robin scheduling for time-varying channels with limited feedback, in 2009 IEEE 10th Workshop on Signal Processing Advances in Wireless Communications, June, pp. 231-234

2. Kuurne A, Miettinen A (2004) Weighted round robin scheduling strategies in (E)GPRS radio interface. In: Proceedings of the IEEE International Vehicular Technology Conference (VTC), vol 5, pp 3155-3159

3. Minelli M, Ma M, Coupechoux M, Godlewski P (2016) Scheduling impact on the performance of relay-enhanced lte-a networks. IEEE Trans Veh Technol 65(4):2496-2508

4. Wong CY, Cheng RS (1999) Multiuser OFDM with adaptive subcarrier, bit, and power allocation. IEEE J. Sel. Areas Commun

5. Bechir Dadi M Belgacem Chibani R (2017), Scheduling performance's study for Ite downlink system. In: 2017 international conference on green energy conversion systems (GECS), March , pp 1-4

6. Viswanath $\mathrm{P}$, Tse DNC, Laroia R (2002) Opportunistic beamforming using dumb antennas. IEEE Trans Inf Theory 48:1277-1294

7. Hamouda H, Kabaou MO, Bouhlel MS (2017), A cross-layer downlink scheduling scheme for balancing qos in ieee 802.16 broadband wireless access systems. In: 2017 IEEE 86th vehicular technology conference (VTC-Fall), pp 1-5

8. Ge X, Jin H, Leung VCM (2016) Cdf-based scheduling algorithm for proportional throughput fairness. IEEE Commun Lett 20(5):1034-1037

9. Masson M, Altman Z, Altman E (2020) Multi-user collaborative scheduling in $5 \mathrm{~g}$ massive mimo heterogeneous networks, in. IFIP Netw Conf 2020:584-588

10. Gueguen C, Baey S (2009), A fair opportunistic access scheme for multiuser OFDM wireless networks, Journal on Wireless Communications and Networking. European Association for Signal Processing (EURASIP). Special issue: Fairness in Radio Resource Management for Wireless Network, Feb

11. Gueguen C, Manini M (2018), Dynamic tradeoff between energy and throughput in wireless $5 \mathrm{G}$ networks. Wirel Commun Mob Comput J, pp 1-12
12. del Olmo MR, Torrea-Duran R, Orozco-Lugo AG, Moonen M (2015) Energy-efficient user scheduling algorithm for Ite networks, in Proc. 5th Joint WIC/IEEE SP Symp. Inf. Theory Signal Process. Benelux (WIC), pp. 1-8

13. Gueguen C (2013) Opportunistic energy aware scheduler for wireless networks. In: Proceedings of the IEEE International 77th vehicular technology conference (VTC Spring), pp 1-5

14. Van Dam T, Langendoen K (2003), An adaptive energy-efficient mac protocol for wireless sensor networks, in Proceedings of the 1st international conference on Embedded networked sensor systems. ACM, , pp. 171-180

15. Kela P, Turkka J, Costa M (2015) Borderless mobility in $5 \mathrm{~g}$ outdoor ultra-dense networks. IEEE Access 3:1462-1476

16. Li YG, Seshadri N, Ariyavisitakul S (1999) Channel estimation for OFDM systems with transmitter diversity in mobile wireless channels. IEEE J Sel Areas Commun 17(3):461-471

17. Truman TE, Brodersen RW (1997) A measurement-based characterization of the time variation of an indoor wireless channel. In: Proceedings of the universal personal communications record (ICUPC), IEEE International

18. Proakis JG, Salehi M (2007) Digital Communications. McGrawHill, New York

19. Tanwir S, Perros H (2013) A survey of vbr video traffic models. IEEE Communications Surveys Tutorials 15(4):1778-1802

20. Tamimi AKA, Jain R, So-In C (2010) Modeling and prediction of high defninition video traffic: A real-world case study. In: 2010 Second international conferences on advances in multimedia, June, pp 168-173

21. Heyman DP (1997) The Gbar source model for vbr videoconferences. IEEE/ACM Trans Netw 5(4):554-560

Publisher's Note Springer Nature remains neutral with regard to jurisdictional claims in published maps and institutional affiliations. 